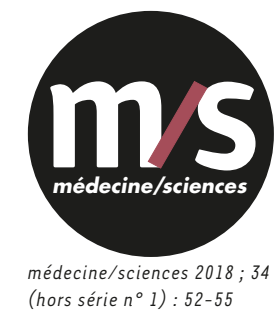

L'action de la France en Europe

et ce que l'Europe peut inspirer

à la France, du point de vue

des malades

Yann Le Cam

> C'est la France qui a initié le règlement européen sur les médicaments orphelins en 1996, sous présidence française de l'Union européenne. C'est Simone Veil, ministre de la Santé, qui l'a introduit, à partir du travail effectué par Annie Wolf dans son ministère. Ce règlement a été adopté en 1999 grâce à son rapporteur Françoise Grossetête. À l'époque, une mission «médicaments orphelins et médicaments pédiatriques » avait été placée entre le cabinet ministériel et les services du ministère de la Santé. II est regrettable que cette bonne méthode n'existe plus pour le Plan national maladies rares.

En 2006, c'est aussi la France qui avait initié un règlement sur l'usage pédiatrique des médicaments et qui l'avait porté. C'est à nouveau Françoise Grossetête qui était rapporteur au Parlement européen. À nouveau, les associations de maladies rares se trouvaient en première ligne pour promouvoir ce règlement, contrairement à ce qui s'est passé aux États-Unis où il s'agissait plutôt des associations contre le cancer et le sida. L'année suivante, a été adopté le règlement sur les thérapies avancées, c'est-à-dire les thérapies géniques et cellulaires. Nous nous sommes battus en première ligne, car les fausses idées reçues étaient nombreuses.

Les associations de patients ont pu promouvoir ces trois règlements grâce au soutien de l'AFM-Téléthon et à ses bénévoles, qui ont financé $100 \%$ de leur action de revendication.

Au cours de cette période, en 1997, la base d'information Orphanet est créée. Il s'agit aujourd'hui de la référence mondiale dans le champ de la connaissance des maladies rares.

Le mouvement européen d'associations de patients Eurordis a été créé la même année en France, par quatre associations françaises; I'AFM-Téléthon, Vaincre la mucoviscidose, AIDES et la Ligue Nationale contre le Cancer. Aujourd'hui, nous regroupons 800 associations dans toute l'Europe.

Je souhaiterais insister sur d'autres éléments. Les États-Unis abordent la problématique des maladies rares sous l'angle du développement des médicaments et de la recherche, et non de la santé publique. La stratégie que nous avons développée en Europe et en France a été inverse. En 2003, nous avons produit un document sur les maladies rares comme outil conceptuel de politique de santé publique. En France, c'est un rapport des États Généraux de la Santé en 1999 que j'ai rédigé pour Bernard Kouchner et un rapport de Bernard Barataud au Conseil économique et

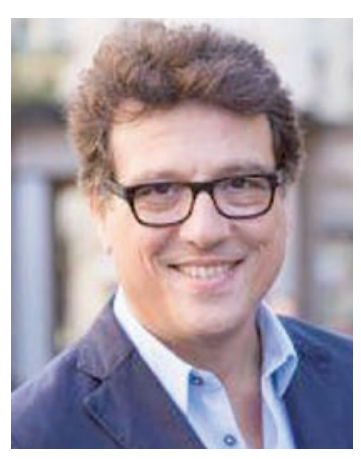

Chief Executive Officer, EURORDIS-Rare Diseases Europe, Plateforme Maladies Rares, 96, rue Didot, 75014 Paris, France.

yann.lecam@eurordis.org

social en 2001, ont posé les bases d'une approche globale sur les maladies rares. C'est cette stratégie, développée d'abord en France avec le premier plan maladies rares de 2005, puis au niveau européen, qui inspire aujourd'hui le Canada et l'Australie, ainsi que des pays d'Amérique du Sud et d'Amérique centrale.

En 2008, la Commission européenne publie une Communication qui fait des maladies rares une priorité de ses différents programmes. Un an plus tard, le Conseil de I'Union Européenne publie une Recommandation fixant, aux États membres, l'objectif de développer des plans maladies rares d'ici 2013. Les plans effectivement adoptés varient dans 25 États membres, mais partagent tous un certain nombre de points communs, qui forment un socle sur lequel il est possible de bâtir : des centres d'expertise, une politique sur les registres, une politique d'investissement dans la recherche et une reconnaissance des patients atteints de maladies rares dans le système.

Le 9 mars 2011, une Directive sur les droits des patients aux soins transfrontaliers est adoptée, composée de quatre éléments clés pour les maladies rares:

- l'affirmation des maladies rares comme une priorité des soins transfrontaliers;

- la création de réseaux européens de référence ;

- l'accès aux soins transfrontaliers ;

- la collaboration européenne pour l'évaluation en santé HTA.

Suite au fort impact du règlement sur les médicaments orphelins, 1300 désignations et plus de 130 médicaments 
avec statut orphelin ont été mis sur le marché et 24 réseaux européens de référence ont été mis en place. Ces réseaux sont formés par groupes thérapeutiques de maladies rares. Leurs missions sont claires et les patients sont impliqués dans la gouvernance. Rien de tout cela n'était évident.

\section{L'implication d'EURORDIS}

La France et les associations de patients ont contribué à la mise en œuvre de ce dispositif, en défendant une vision politique et humaine plus que bureaucratique. En d'autres termes, il s'agit de définir des chemins thérapeutiques centrés sur les patients, où chacun d'entre eux trouverait sa place. L'objectif est également de réduire les inégalités entre territoires et entre maladies rares et de faire voyager l'expertise plus que les patients.

Au moment de la mise en œuvre par l'Union Européenne, nous avons décidé de devenir prestataires de la Commission européenne dans un appel d'offres aux côtés de l'organisation européenne des hôpitaux et de l'agence d'accréditation canadienne des hôpitaux, de manière à forger le processus de soumission et d'évaluation de ces réseaux.

II s'agissait du seul moyen de faire en sorte que ces réseaux et soient centrés sur l'excellence clinique et l'amélioration (patient health outcome improvement) ne se cantonnent pas à un rôle de connexion administrative. Nous avons été extrêmement soutenus par de nombreux représentants d'États membres et les académiques, dont Ségolène Aymé qu'il faut remercier.

L'axe collaboratif a guidé notre action. Nous avons ainsi évité une catastrophe. Les responsables européens et certains États membres souhaitaient lancer un appel d'offres ouvert, compétitigs entre réseaux d'une même maladie mais nous avons privilégié la collaboration au détriment de la compétition. Nous y sommes parvenus, grâce à des initiatives en amont, comme Orphanet et son système d'Orphacodes qui a permis de structurer les regroupements thérapeutiques et l'Action Conjointe Maladies Rares (JA RD Actions). En France, l'expérience des centres de référence et des filières de soins ont permis de définir des regroupements. L'expérience encore plus ancienne du Royaume-Uni s'est également avérée précieuse. Enfin, des tendances émergeaient en Allemagne. Tout cela a été consolidé pour définir ces 24 réseaux européens de référence entre 960 centres experts.

Ces réseaux sont conçus pour appliquer partout en Europe nos acquis de connaissances en termes de prise en charge des maladies rares, et générer de nouvelles connaissances. Pour cette raison, parmi les critères de sélection, figure leur participation à des travaux de recherche. L'enjeu est aujourd'hui de lier les soins, la recherche et les données.

En réalité, c'est le même patient qui se rend dans le même centre hospitalier et qui parle à peu près aux mêmes médecins. Les mêmes compétences sont requises pour appliquer les bonnes pratiques de soins pour réaliser un essai clinique ou collecter les données après l'approbation de la mise sur le marché du produit.

Trois niveaux d'innovation sont requis, à savoir un niveau organisationnel, un niveau scientifique et un niveau technologique.
Il faut aussi décloisonner le public et le privé, ce qui ne fonctionne pas bien aujourd'hui. Nous essayons de structurer les discussions entre industriels et pouvoirs publics nationaux et européens, afin de mettre en place un cadre de collaboration dans la collecte des données, l'histoire naturelle des maladies, les registres, la mise en place d'essais cliniques et la collecte de données réelles après mise sur le marché. Certains États membres sont toutefois pleins de fantasmes, craignant une collaboration avec les «méchants industriels ».

II nous faut pourtant avancer dans ce cadre vers des données portant sur le plus grand nombre de patients possible, de qualité, standardisées correspondant aux exigences réglementaires et disponibles le moment voulu. II s'agit d'un changement possible dans la relation entre industriels et réseaux de soins, grâce à ces réseaux européens. Sans doute faudra-t-il définir un cadre juridique et prévoir davantage de financement.

Cette stratégie et ces opportunités nous conduisent à une véritable transformation. C'est une chance inouïe, par rapport à dix ans auparavant. II faut se saisir de cette chance et en mesurer tout le potentiel. Au cœur de ces transformations, la co-construction entre États membres, cliniciens, gestionnaires d'hôpitaux et patients et entre secteurs public et privé est rendue possible grâce à l'échelon européen. J'espère que le débat européen viendra nourrir le débat en France.

La collaboration en matière de recherche est essentielle. Nous avons aujourd'hui l'opportunité de travailler de manière structurée et cohérente pour l'avenir.

\section{Une grande ambition pour les 10 prochaines années}

Nous devons avoir une grande ambition pour les dix prochaines années, et pour la façon dont la France peut jouer un rôle. La ministre de la Santé exprime une volonté politique forte dans ce domaine. Elle n'a plus qu'à traduire sa volonté en acte pour être à la hauteur de Simone Veil.

II n'y aura de troisième plan maladies rares ambitieux en France que s'il se place dans une perspective européenne, voire internationale. L'un ne s'oppose pas l'autre. L'un nourrit l'autre.

En France, il faut assumer le fait que notre pays a joué un rôle leader dans le champ des maladies rares. II faut croire dans le rôle leader que nous pouvons jouer ces prochaines années. En matière de génétique, nous sommes à la traîne et tentons de rattraper le retard. Dans le champ des maladies rares en revanche, nous faisons mieux que les autres. Profitons-en et inspirons le niveau européen. 
Le projet de troisième plan s'avère fort intéressant. II doit être innovant sur les questions de données et de santé connectée. Le plan doit se dérouler sur une période de dix ans. Ce plan a donc un rôle d'impulsion fondamental pour les prochaines années.

Au niveau européen, nous pouvons avoir une nouvelle ambition, dont la France peut être le vecteur. Celle-ci peut inspirer une politique de collaboration renforcée en matière de recherche, d'organisation des soins, de collecte et d'analyse de données, de développement et d'accès aux médicaments.

Cette ambition doit se traduire en objectifs. Pouvoir être diagnostiqué pour sa maladie rare en moins d'un an est un objectif partagé, qui semble raisonnable. Ceux qui n'ont pas de diagnostic doivent être tous inclus dans des programmes de recherche.

Un autre objectif porte sur les nouveaux traitements. Plus de 300 ont été approuvés au cours des sept dernières années. On pense que 600 à 1000 autres seront développés et approuvés dans les dix ans à venir. II existe un potentiel énorme issu de la science, qui peut être traduit en thérapies nouvelles pour répondre aux besoins des malades.

Concernant le médicament, je ne résiste pas à l'envie d'évoquer la stratégie d'Eurordis industriels, des payeurs et des agences d'évaluation. Sur notre site, un document en anglais de 70 pages formule un grand nombre de propositions. Notre objectif est simple : cinq fois plus de médicaments dans dix ans, mais cinq fois moins chers. On nous rétorque que nous sommes des utopistes, mais nous ne le sommes pas plus que lorsque nous avons débuté, en 1997. Arrêtons de discuter d'économies de $10 \%$ et de restriction de l'accès aux soins aux patients. Ce qui compte est de développer des thérapies et de faire en sorte que les patients y aient ensuite accès. II faut complètement changer de modèle. II n'existe pas de solution magique mais un ensemble de leviers qu'il faut actionner.

D'autres stratégies de R\&D et de méthodes d'essais cliniques peuvent être utilisées. De nombreuses alternatives sont déjà encouragées et soutenues par l'Agence européenne du médicament ou les agences d'évaluation de santé. Se développe également l'analyse de l'utilité d'un médicament du point de vue du patient. D'autres éléments permettent de réduire fortement les coûts.

Hier, développer une agence réglementaire européenne émettant des avis communs semblait une utopie. Aujourd'hui, c'est notre quotidien. Il y a cinq ans, nous disions qu'il était utopique de faire collaborer les agences type HAS au niveau européen. Pourtant, c'est pour demain. Commençons donc à parler de la collaboration entre les payeurs. Pour les maladies rares, il est insensé de continuer à travailler au niveau national. Il faut s'asseoir autour d'une table avec les autres pour renforcer le pouvoir de négociation, engager un dialogue et innover sur les schémas d'accès et financement. Quelle compagnie refusera d'entrer sur le marché européen? Aucune. Rééquilibrons les pouvoirs, non pour taper sur la tête de l'industriel, mais pour parler économique et scientifique dans le cadre de la même discussion. Arrêtons de séparer les choses, puisque le sujet est le même.

Le problème des incertitudes lors de la mise sur le marché doit être réduit par des recherches adaptées. II n'y a aucune raison de payer cher pour des médicaments incertains. II faut des modèles dans le cadre desquels l'industriel fait payer moins cher, et où les pouvoirs publics investissent aux côtés des industriels le temps de générer des données. Différentes approches sont possibles. Il suffit d'ouvrir ce dialogue et de se lancer avec audace.

J'espère que, dans dix ans, nous constaterons que la table de négociations européennes sur les médicaments fonctionne, qu'il existe des appels d'offres communs et des négociations communes. L'argent sera toujours collecté par les cotisations locales. Cela n'empêche pas de s'asseoir à la table européenne des négociations et de prendre ensuite une décision nationale. Essayons de faire mieux, avec le même argent. Donnons de la visibilité économique aux entreprises, aux investisseurs et aux payeurs.

Au niveau européen, nous utilisons les présidences européennes. Ainsi, Malte a joué un rôle clé pour relancer la dynamique sur les maladies rares. La conclusion du Conseil des ministres de juin 2017 est d'établir un rapport sur l'application de la Communication de 2008 et de la Recommandation du Conseil de 2009, de manière à éventuellement relancer une nouvelle législation sur les maladies rares. Nous travaillons déjà avec la présidence autrichienne de 2018 et la présidence roumaine de 2019, afin de monter en puissance. Nous espérons être capables de mettre à l'ordre du jour une nouvelle loi européenne permettant d'accélérer l'intégration dans certains domaines clés.

Nous avons également mis en place un réseau européen parlementaire composé de députés européens, qui associera également des parlementaires nationaux de différents pays de l'Union européenne.

Nous espérons que la France soutiendra les collaborations renforcées en matière d'évaluation des technologies en santé dans le cadre de la législation européenne à venir, et qu'elle se portera candidate pour accueillir le secrétariat scientifique permanent.

Nous espérons que la France continuera à soutenir la coopération renforcée en matière de recherche et une nouvelle législation sur les maladies rares en 2019-2020.

\section{Des initiatives internationales}

Enfin, dans le cadre de l'ONU, nous profitons du nouveau calendrier 2015-2030 des Objectifs de Développement Durable, pour positionner les maladies rares. Nous avons créé un comité des ONG sur les maladies rares à New York. Nous commençons à travailler avec différents États membres. Un chemin est possible pour aller vers une Résolution des Nations Unies sur les 
maladies rares. La France, qui est I'un des pays les plus influents au Conseil de sécurité et au Conseil économique et social, saura, nous l'espérons, porter l'action qu'elle entreprend depuis vingt ans au niveau international. N'abandonnons pas ceux qui ont moins de chance ailleurs. Nous nous en porterons tous mieux, y compris en France. $\diamond$
French model for Europe and Europe as a model for France in the field of rare diseases

\section{LIENS D'INTÉRÊT}

L'auteur déclare n'avoir aucun lien d'intérêt concernant les données publiées dans cet article.

\section{La France dans la recherche européenne et internationale sur les maladies rares}

Daria Julkowska

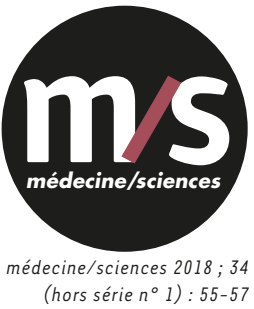

> L'objectif de cette présentation est de positionner la France dans la recherche européenne et internationale sur les maladies rares, ses réussites et ses perspectives. Je souhaiterais également évoquer l'importance de la collaboration internationale pour aujourd'hui et demain.

Il existe différentes organisations œuvrant dans le domaine de la recherche sur les maladies rares. L'IRDiRC est un consortium international de recherche sur les maladies rares, chargé d'établir une stratégie de financement de la recherche, des guidelines et des recommandations en matière de recherche.

$\varepsilon$-Rare et la Commission européenne financent la recherche sur les maladies rares. Les réseaux européens de référence constituent également une source d'expertise en matière de recherche. Les associations de patients œuvrent par le biais d'Eurordis. II existe par ailleurs de nombreuses infrastructures européennes, comme Orphanet, Elixir et le Joint Research Center de la Commission Européenne. Nous souhaitons aujourd'hui intégrer l'activité de l'ensemble de ces entités sous un seul chapeau.

La France est partenaire de l'IRDiRC depuis ses débuts, par le biais de l'Agence Nationale de la Recherche, la fondation maladies rares et AFM, mais également parce que la France fournit le secrétariat scientifique de l'IRDiRC, porté par l'Inserm et financé par la Commission Européenne.

La France est intégrée aux réseaux européens de référence et coordonne cinq d'entre eux. Elle se trouve également impliquée dans la majorité des infrastructures européennes médicales. Orphanet a été créé et porté par la France, ainsi qu'Eurordis. Depuis les débuts, la France a coordonné un programme d'importance, l'ERA-Net « $\varepsilon$-Rare», qui regroupe des agences de financement de multiples pays.

La France ne dispose pas d'un programme spécifique de financement dédié à la recherche sur les maladies rares. II existe toutefois des

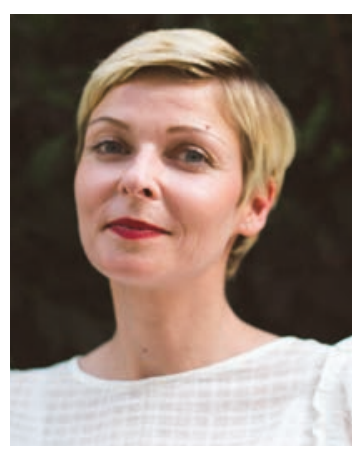

Coordinateur scientifique, Agence Nationale de Recherche, 50 , avenue Daumesnil, 75012 Paris, France. daria.julkowska@ agencerecherche.fr

financements par le biais de l'ANR, qui finance des projets sur les maladies rares via son appel à projets générique, entre 8 et 15 millions d'euros par an. Dans le cadre d'autres plans, la France investit entre 15 et 20 millions d'euros par an.

La DGOS soutient également la recherche et le soin, avec plus de 20 millions d'euros d'investissements par an. D'autres organismes sont d'importance, comme I'AFM et la fondation maladies rares.

\section{Le programme de financement de la recherche $\varepsilon$-Rare}

$\varepsilon$-Rare est un programme de financement de la recherche qui a été créé en 2006. II a été coordonné par l'Inserm entre 2006 et 2014 . Entre 2014 et 2019, il est coordonné par l'ANR.

Aujourd'hui, le consortium regroupe 28 agences de financement de 20 pays européens, associés et non européens (Figure 1). Au fil des ans, nous avons recruté de nouveaux pays, comme le Canada, le Japon, Israël et la Turquie. Son objectif principal est de coordonner et de promouvoir la 